\title{
Check-list de Asteraceae no estado de Mato Grosso do Sul, Brasil
}

\author{
Nádia Roque', Aristônio M. Teles², Lúcia Moura', Rodrigo Andrade Pacheco³, Gustavo Henrique \\ Lima da Silva ${ }^{2}$, Maria Alves ${ }^{4}$ \& Jimi Naoki Nakajima ${ }^{3}$
}

\author{
'Universidade Federal da Bahia, Instituto de Biologia, Av. Ademar de Barros, s/n., Campus Universitário de Ondina, \\ CEP 40.170-110, Salvador, Bahia, Brasil. nadiaroque@gmail.com \\ 2Universidade Federal de Goiás, Instituto de Ciências Biológicas, Departamento de Botânica, Campus Samambaia, saída para Nerópolis \\ - km 13. CEP 74.690-900, Goiânia, Goiás, Brasil. \\ 3Universidade Federal de Uberlândia Instituto de Biologia. CEP 38.400-902, Uberlândia, Minas Gerais, Brasil. \\ ${ }^{4}$ Universidade Estadual de Feira de Santana, Departamento de Ciências Biológicas, Programa de Pós-Graduação em Botânica, \\ BR $116, \mathrm{~km}$ 03, CEP 44.031-460, Feira de Santana, Bahia, Brasil.
}

Recebido em 27.XI.2014

Aceito em 10.IX.2015

DOI 10.21826/2446-8231201873s147

RESUMO - O check-list da família Asteraceae no Mato Grosso do Sul é apresentado visando contribuir para ações de conservação no estado. Para a confecção da listagem foram utilizados os bancos de dados e os levantamentos florísticos realizados. Asteraceae está representada por 16 tribos, 99 gêneros e 343 espécies, e dentre as 32 espécies reconhecidas como endêmicas para o estado, 11 são restritas às áreas do Pantanal. Os gêneros com maior riqueza foram Baccharis L. (24 espécies), Chromolaena DC. (22 spp.) e Lessingianthus H. Rob. (22 spp.). Os gêneros que se destacam pela elevada representatividade em áreas de Mata Atlântica, como Baccharis L. e Mikania Willd., estão provavelmente subamostrados no estado. Com relação ao Pantanal, o elevado número de espécies endêmicas revela sua significância ecológica e confirma a prioridade para conservação desse ecossistema.

Palavras-chave: Compositae, flora do Brasil, inventário florístico

ABSTRACT - Checklist of Asteraceae in Mato Grosso do Sul state, Brazil. This paper aims to produce a checklist of the family Asteraceae in Mato Grosso do Sul and to subsidize conservation action in the state. To make this checklist all databases and floristic inventories carried out in Mato Grosso do Sul were verified. Asteraceae is represented by 16 tribes, 99 genera and 343 species. From 32 species known to be endemic to the state, 11 are restricted to the Pantanal. The genera with the largest number of species are Baccharis L. (24 species), Chromolaena DC. (22 spp.) and Lessingianthus H. Rob. (22 spp.). The genera with higher representation in the Atlantic Forest, such as Baccharis L. and Mikania Willd., are probably undersampled in the state. With regards to the Pantanal, the greater number of endemic species reveals its ecological significance and confirms the priority for conservation of this ecosystem.

Keywords: Compositae, flora of Brazil, floristic inventory

\section{INTRODUÇÃO}

Asteraceae é a maior família dentre as Angiospermas, com aproximadamente 24.000 espécies, agrupadas em 1.600-1700 gêneros, o que representa cerca de $10 \%$ das Angiospermas (Funk et al. 2009). O grupo tem distribuição cosmopolita e maior abundância nas regiões temperadas e semiáridas dos trópicos e subtrópicos. A família caracterizase pela inflorescência em capítulo, circundado por brácteas involucrais, anteras sinânteras, com exposição secundária do grão de pólen, ovário ínfero, bicarpelar, unilocular, que se desenvolve em uma cipsela geralmente com pápus (Roque \& Bautista 2008, Funk et al. 2009).

Recentemente Panero \& Funk (2008) e Funk et al. (2009), a partir da análise filogenética utilizando diversos marcadores moleculares, reconheceram para a família 12 subfamílias e 43 tribos, das quais 28 ocorrem no Brasil. Segundo BFG (2015), são registrados para o Brasil, 278 gêneros e 2.084 espécies, representados em todos os biomas, porém com maior diversidade nas formações campestres, como cerrado, campos rupestres e campos sulinos, e menos frequentes em áreas de restinga, caatinga, brejos e florestas de altitude (Hind \& Miranda 2008). No Brasil, Eupatorieae é a maior tribo de Asteraceae em número de gêneros (86) e espécies (615), seguido por Vernonieae (51 gêneros, 465 espécies), Heliantheae (32/234), Astereae (20/244) e Senecioneae (8/95).

Uma vez que os inventários florísticos proporcionam informações essenciais para o entendimento da distribuição geográfica e dos centros de diversidade, para o estabelecimento do manejo de áreas protegidas e propor medidas de conservação (IUCN 2010), o checklist das espécies de Asteraceae do estado de Mato Grosso do Sul tem como foco a melhoria do conhecimento disponível sobre a flora do estado, o que implicará em um diagnóstico 
sobre as fitofisionomias existentes, agregando informações para subsidiar a conservação de áreas prioritárias.

\section{Principais Grupos de Pesquisa}

Dentre os principais grupos de pesquisa de Asteraceae, cabe destacar o grupo criado em 2006 e intitulado "Estudos multidisciplinares em Asteraceae" com a participação de 23 pesquisadores, 26 alunos e um biólogo. Este grupo tem formado alunos e participado da produção acadêmica significativa sobre a família no país.

Contudo, cabe ressaltar, que a equipe que participou da Lista da Flora do Brasil (BFG 2015) representa uma parcela significativa das gerações de sinanterólogos no país e que tem se unido pelo desenvolvimento de dados sobre a família em diversas linhas de pesquisa e cujos produtos têm gerado impactos de relevância nacional e internacional sobre a flora do país.

\section{Principais Lacunas de Conhecimento}

Em relação à listagem de espécies de Asteraceae para o Mato Grosso do Sul, os gêneros que se destacam pela elevada representatividade em áreas de Mata Atlântica, como Baccharis L. (113 espécies) e Mikania Willd. (122 espécies) (sensu Teles et al. 2009), estão provavelmente subamostrados em Mato Grosso do Sul. Com relação ao Cerrado, originalmente o bioma mais difundido no estado, observou-se que gêneros amplamente distribuídos, como Lessingianthus H. Rob. e Chromolaena DC. (53 e 27 espécies respectivamente, sensu Mendonça et al. 2008), apresentaram uma significativa riqueza no estado (21 e 22 espécies cada).

Portanto, em se tratando da Flora de Asteraceae para o Mato Grosso do Sul, o aumento no esforço de coleta, principalmente nos domínios de Mata Atlântica e Cerrado, poderá incrementar a diversidade da família no estado. Com relação à flora da família relacionada ao Pantanal, o elevado número de espécies endêmicas (11 espécies) revela sua significância ecológica e confirma a prioridade para conservação desse ecossistema.

\section{MATERIAL E MÉTODOS}

Para a confecção da lista de espécies foram utilizadas, como ponto de partida, as espécies citadas para o estado de Mato Grosso do Sul na Lista do Brasil (BFG 2015). A seguir foram acrescentadas as espécies citadas no CRIA (2017) e no FloResCer (2017), e cuja identificação tenha sido feita por algum especialista da família, como por exemplo, Graziela M. Barroso, Roberto Esteves, Nicholas Hind, Harold Robinson, entre outros. Além disso, foi consultada a lista para a flora do Mato Grosso (Dubs 1998) e realizadas buscas, na base de dados do SciELO (Scientific Eletronic Library Online) e Biological Abstracts, de inventários florísticos realizados no estado nos últimos dez anos.

Para a citação dos vouchers foi utilizada a ordem de prioridade de: material-tipo e espécimes citados em protólogos, revisões (e.g. Blake 1921, Sherff 1932, 1937,
Cabrera 1957, 1971, Powell 1965, Stuessy 1972, Barroso 1976, Canne 1977, Jansen 1981, 1985, Bautista 1986, Bolick 1991, Urtubey 1999, Santos 2001, Moraes \& Semir 2009), teses e dissertações na área de taxonomia vegetal (e.g. Pereira 2001, Mondin 2004, Magenta 2006, Bringel Jr. 2007, Deble 2007, Saavedra 2011), lista de espécies obtida de Dubs (1998) e as citações no CRIA (2017), cuja identificação se deu por especialistas na família. Os trabalhos gerais (biologia floral, levantamento florístico, interação inseto-planta, etnobotânica, etc.) envolvendo a flora do Mato Grosso do Sul foram analisados para confirmação (ou não) da citação do táxon para o estado.

Deste checklist foram excluídas 50 espécies citadas para o Mato Grosso do Sul (Lista da Flora do Brasil, listas florísticas regionais e publicações em geral), uma vez que os vouchers não foram localizados para o estado. Deste total, 15 espécies pertenceram à tribo Vernonieae, 15 à tribo Eupatorieae, seis espécies à tribo Astereae, sete à Heliantheae e sete táxons referiram-se às demais tribos.

\section{RESULTADOS E DISCUSSÃO}

A lista de Asteraceae para o estado de Mato Grosso do Sul está representada por 16 tribos, 99 gêneros e 338 espécies, sendo que as maiores tribos são Eupatorieae (28 gêneros e 94 espécies), Heliantheae (20/67), Vernonieae $(18 / 66)$ e Astereae (6/31), seguindo a tendência na representatividade das regiões centrais do país (Quadro 1). As tribos basais (Barnadesieae, Mutisieae, Onoserideae, Nassauvineae e Gochnatieae) contêm o menor número de representantes no estado, com até dois gêneros cada, totalizando 16 espécies.

Segundo BFG (2015), há um gênero e 32 espécies endêmicos para o Mato Grosso do Sul e das quais, 11 são restritos ao Pantanal (Aspilia silphioides (Hook. \& Arn.) Benth. \& Hook., Calea rupicola Chodat, Dimerostemma annuum (Hassl.) H. Rob., Dimerostemma apense (Chodat) M.D. Moraes, Dimerostemma aspilioides (Griseb.) M.D. Moraes, Dimerostemma virgosum H. Rob., Disynaphia achillaea (Chodat) R.M. King \& H. Rob., Lycoseris boliviana Britton, Flaveria bidentis (L.) Kuntze, Mikania stenophylla W.C. Holmes e Wedelia brachylepis Griseb.).

Os gêneros com maior número de espécies foram Baccharis L. (24 espécies), Chromolaena DC. (22 spp.) e Lessingianthus H. Rob. (21 spp.), seguidos por Aspilia Thouars (15 spp.), Calea L. (15 spp.) e Dimerostemma Cass., Mikania Willd. e Vernonanthura H. Rob. (14 spp. cada). Estes valores são pouco expressivos quando comparado ao elevado número de espécies destes gêneros referidos aos biomas de Cerrado (Mendonça et al. 2008) e Mata Atlântica (Teles et al. 2009), fitofisionomias originalmente dominantes no estado.

Segundo BFG (2015), dentre as espécies de Asteraceae listadas como raras no Brasil, cinco delas são provenientes do Mato Grosso do Sul, Aspilia grazielae J.U. Santos, $A$. hatschbachii J.U. Santos, Ichthyothere matogrossensis R.C.A. Pereira \& Semir e Vernonia gertii Dematteis. 
Quadro 1. Lista das espécies de Asteraceae registradas para o estado do Mato Grosso do Sul, com seus respectivos coletores e herbários depositários.

\begin{tabular}{|c|c|}
\hline Espécies & Voucher \\
\hline Acanthospermum australe (Loefl.) Kuntze & Hatschbach 21867, MBM \\
\hline A. hispidum DC. & Hatschbach 21867, RB \\
\hline Achyrocline flaccida (Weinm.) DC. & Equipe Santa Inês, RB 316532 \\
\hline A.gertiana Deble \& Marchiori & Chagas \& Silva 767, holótipo MBM \\
\hline A. satureioides (Lam.) DC. & Malme 1282, S \\
\hline Acilepidopsis echitifolia (Mart. ex DC.) H. Rob. & Hatschbach 33804, MBM \\
\hline Acmella alpestris (Griseb.) R.K. Jansen & Hatschbach 74220, MBM \\
\hline A. bellidioides (Smith in Ress) R.K. Jansen & Hatschbach 25140, RB \\
\hline A. decumbens (Sm.) R.K. Jansen & Hatschbach \& Barbosa 76331, HUCS \\
\hline A. grisea (Chodat) R.K. Jansen & Hatschbach 76212, MBM \\
\hline A. leptophylla (DC.) R.K. Jansen & Hatschbach et al. 74578, ESA \\
\hline A. oleracea (L.) R.K. Jansen & Cunha 331, CGMS \\
\hline Adenostemma suffruticosum Gardner & Hatschbach 24351, MBM, S, Z \\
\hline Ageratum conyzoides L. & Malme 1644, S \\
\hline Aldama bracteata (Gardner) E.E. Schill. \& Panero & Heringer 11407, RB \\
\hline A. corumbensis (Malme) Magenta \& Pirani & Hatschbach et al. 74900, MBM, SPF \\
\hline A. grandiflora (Gardner) E.E. Schill. \& Panero & Pott \& Pott 8137, CPAP, SPF \\
\hline A. linearifolia (Chotat) E.E. Schill. \& Panero & Hatschbach et al. 58730, MBM \\
\hline A. macrorhiza (Baker) E.E. Schill. \& Panero & Gomes 2397, SP \\
\hline A. pilicaulis (S.F.Blake) E.E. Schill. \& Panero & Hatschbach et al. 74393, MBM, SPF \\
\hline A. rubra (Magenta \& Pirani) E.E. Schill. \& Panero & Caliente 298, HISA, UEC \\
\hline A. squalida (S. Moore) E.E. Schill. \& Panero & Vasconcelos et al. 25, CGMS \\
\hline A. tuberosa (Griseb.) E.E. Schill. \& Panero & Sugiyama \& Pinto 139, CGMS \\
\hline A. weddellii (S.F.Blake) E.E. Schill. \& Panero & Hatschbach 25020, MBM, RB \\
\hline Alomiella regnellii (Malme) R.M. King \& H. Rob. & Malme 1678, holótipo S \\
\hline Ambrosia artemisaefolia $L$. & Souza 1783, HUEM 19115 \\
\hline Aspilia attenuata (Gardner) Baker & Hatschbach 73298, UB \\
\hline A. camporum Chodat & Santos s.n., R 36961 \\
\hline A. clausseniana Baker & Hatschbach 45962, MBM \\
\hline A. elata Pilg. & Pott $1115, \mathrm{RB}$ \\
\hline A. floribunda (Gardner) Baker & Silva 185, SP \\
\hline A. foliacea (Spreng.) Baker & Emygdio $2050, \mathrm{R}$ \\
\hline A. grazielae J.U. Santos & Barroso s.n., holótipo RB 117472; Isótipo HB 26280 \\
\hline A. hatschbachii J.U. Santos & Hatschbach et al. 35983, holótipo MBM \\
\hline A. heringeriana H. Rob. & Hatschbach et al. 76546, BHCB \\
\hline A. latíssima Malme & Paula et al. 3190, MG \\
\hline A. leucoglossa Malme & Hatschbach 45900, MBM \\
\hline A. montevidensis (Spreng.) Kuntze & Pereira et al. 241, RB \\
\hline A. platyphylla (Baker) S.F. Blake & Hatschbach 24623, RB \\
\hline A. reflexa (Sch.Bip. ex Baker) Baker & Magenta 685, SPF \\
\hline A. silphioides Benth. \& Hook.f. & Silva 127, SP \\
\hline Austroeupatorium inulifolium (Kunth) R.M. King \& H. Rob. & Hatschbach 77036, MBM \\
\hline Ayapana amygdalina (Lam.) R.M. King \& H. Rob. & Hatschbach 26042, MBM, Z \\
\hline Ayapanopsis esperanzae (Hassl.) R.M. King \& H. Rob. & Malme $1860, \mathrm{~S}$ \\
\hline Baccharis brevifolia DC. & Conceição 2483, RB \\
\hline B. breviseta DC. & Hatschbach 74491, HUEFS \\
\hline B. cognata DC. & Hatschbach 23599, MBM \\
\hline B. crispa Spreng. & Palhano 54, CGMS \\
\hline B. dentata (Vell.) G.M. Barroso & da Silva 4, CGMS \\
\hline B. dracunculifolia DC. & Resende 89, RB \\
\hline B. erigeroides DC. & Conceição 2442, CGMS \\
\hline B. glutinosa Pers. & Hatschbach 22026, RB \\
\hline B. humilis Sch.Bip. ex Baker & Archer s.n., UB 22334 \\
\hline B. illinitoides Malag. & Cordeiro 3057, MBM \\
\hline B. linearifolia (Lam.) Pers. & Hatschbach 46229, MBM \\
\hline B. malmei Joch. Müll. & Aquino 1, CGMS \\
\hline B. notosergila Griseb. & Malme 2783, S \\
\hline
\end{tabular}


Quadro 1. Cont.

\begin{tabular}{|c|c|}
\hline Espécies & Voucher \\
\hline B. pingraea $\mathrm{DC}$. & Hatschbach 26026, NY \\
\hline B. punctulata DC. & Silva 4821, FURB \\
\hline B. reticularia DC. & Seramim 133, RB \\
\hline B. retusa DC. & Neves 7, CGMS \\
\hline B. salicifolia (Ruiz \& Pav.) Pers. & Silva \& Barbosa 4980, HUCS \\
\hline B. serrulata (Lam.) Pers. & Moore 1004, BM \\
\hline B. sessiliflora Vahl & Hatschbach 33033, RB \\
\hline B. subdentata DC. & Hatschbach $33102, \mathrm{RB}$ \\
\hline B. tridentata Vahl & Hatschbach 23599, RB \\
\hline B. trinervis Pers. & Damasceno Jr. 01, RB \\
\hline B. vulneraria Baker & Hatschbach et al. 74491, ESA \\
\hline Baltimora geminate (Brandegee) Stuessy & A. Pott \& V.J. Pott 2599, RB \\
\hline Barnadesia caryophylla (Vell.) S.F. Blake & Lorenzi 2189, HPL \\
\hline Barrosoa betoniciformis (DC.) R.M. King \& H. Rob. & Sucre $10520, \mathrm{RB}$ \\
\hline B. candolleana (Hook. \& Arn.) R.M. King \& H. Rob. & Malme 2707, S \\
\hline Bejaranoa balansae (Hieron.) R.M. King \& H. Rob. & Hatschbach 49007, MBM \\
\hline Bidens fistulosa Sch.Bip. ex Baker & Hatschbach 35060, MBM, Z \\
\hline B. gardneri Baker & Ribas \& Pereira 2409, ESA \\
\hline B. pilosa $\mathrm{L}$. & Pereira 268, RB \\
\hline B. riedelii Baker & Hatschbach 26038, MBM, NY \\
\hline B. segetum Mart. ex Colla & Caliente 76 , HISA \\
\hline Brickellia difusa (Vahl) A.Gray & Silva 4947, ALCB \\
\hline Calea anomala Hassl. & Hatschbach 34014, MBM, Z \\
\hline C. chapadensis Malme & Malme 2123, S \\
\hline C. clausseniana var. riedeliana Baker & Riedel 667, Isótipo, NY \\
\hline C. clematidea Baker & Hatschbach 73274, MBM \\
\hline C. crenata Chodat & Hatschbach 48405, MBM \\
\hline C. cuneifolia DC. & Hatschbach 25147, MBM \\
\hline C. cymosa Less. & Hatschbach et al. 76674, ESA, MBM \\
\hline C. ferruginea Sch.Bip. ex Baker & Malme 1416, S \\
\hline C. lantanoides Gardner & Barbosa \& Silva 1462, ALCB, SPF \\
\hline C. mediterranea (Vell.) Pruski & Hatschbach $25223, \mathrm{~S}$ \\
\hline C. reticulate Gardner & Hatschbach 25079, MBM \\
\hline C. rupicola Chodat & Hatschbach 58891, ESA, MBM \\
\hline C. teucriifolia (Gardner) Baker & Hatschbach 35986, MBM \\
\hline C. uniflora Less. & Hatschbach et al 76993, ESA, MBM \\
\hline C. verticillata (Klatt) Pruski & Hatschbach 60666, ESA, MBM \\
\hline Campovassouria bupleurifolia (DC.) R.M. King \& H. Rob. & Pederson 14728, MBM \\
\hline C. cruciate (Vell.) R.M. King \& H. Rob. & Barbosa 1798, RB \\
\hline Campuloclinium macrocephalum (Less.) DC. & Robert 871, BM \\
\hline C. megacephalum (Mart. ex Baker) R.M. King \& H. Rob. & Hatschbach $34171, \mathrm{Z}$ \\
\hline C. riedelii (Baker) R.M. King \& H. Rob. & Hatschbach 25142, MBM \\
\hline Centratherum punctatum Cass. & Malme 3012, S \\
\hline Chaptalia integérrima (Vell.) Burkart & Hatschbach 74177, MBM \\
\hline C. nutans (L.) Pol. & Schardong 328, UPCB \\
\hline Chresta exsucca DC. & Malme 1708, S \\
\hline C. sphaerocephala $\mathrm{DC}$. & Ratter 22, $\mathrm{K}$ \\
\hline Chromolaena arnottiana (Griseb.) R.M. King \& H. Rob. & Hatschbach 77169, MBM \\
\hline C. callilepis (Sch.Bip. ex Baker) R.M. King \& H. Rob. & Amador 49, CGMS \\
\hline C. chaseae (B.L.Rob.) R.M. King \& H. Rob. & Hatschbach 62184, MBM \\
\hline C. christieana (Baker) R.M. King \& H. Rob. & Hatschbach 49125, MBM, Z \\
\hline C. densiflora (Morong.) R.M. King. \& H. Rob. & Ribas 2505, MBM \\
\hline C. ferruginea (Garder) R.M. King. \& H. Rob. & Hatschbach 29420, MBM \\
\hline C. hirsuta (Hook. \& Arn.) R.M. King \& H. Rob. & Hanke 540, FUEL \\
\hline C. horminoides DC. & Irwin $16597, \mathrm{Z}$ \\
\hline C. ivifolia (L.) R.M. King \& H. Rob. & Hatschbach 21949, MBM \\
\hline C. laevigata (Lam.) R.M. King \& H. Rob. & Malme 1542, S, Z \\
\hline C. leucocephala Gardner & Malme 2031, S \\
\hline
\end{tabular}


Quadro 1. Cont.

\begin{tabular}{|c|c|}
\hline Espécies & Voucher \\
\hline C. lilacina (Hieron.) R.M. King \& H. Rob. & Cervi 3261, RB \\
\hline C. margaritensis (Hassl.) R.M. King \& H. Rob. & Malme 2793, S \\
\hline C. maximilianii (Schrad. ex DC.) R.M. King \& H. Rob. & Malme $3410, \mathrm{~S}$ \\
\hline C. odorata (L.) R.M. King \& H. Rob. & Harley $10916, \mathrm{~K}$ \\
\hline C. orbignyana (Klatt) R. M. King. \& H. Rob. & Hatschbach 74399, MBM \\
\hline C. pedunculosa (Hook. \& Arn.) R.M. King \& H. Rob. & Hatschbach 24319, MBM, Z \\
\hline C. pungens (Gardn.) R.M. King \& H. Rob. & Cid Ferreira 2069, RB \\
\hline C. squalida (DC.) R.M. King \& H. Rob. & Hatschbach 21807, MBM \\
\hline C. squarroso-ramosa (Hieron.) R.M. King \& H. Rob. & Malme 1718, S \\
\hline C. squarrulosa (Hook. \& Arn.) R.M. King \& H. Rob. & Hatschbach 49328, MBM \\
\hline C. stachyophylla (Spreng.) R.M. King \& H. Rob. & Hatschbach 58735, MBM \\
\hline Chrysolaena cognata (Less.) Dematt. & Hatschbach 48549, MBM, Z \\
\hline C. desertorum (Mart. ex DC.) Dematt. & Malme 2510, S \\
\hline C. lithospermifolia (Hieron.) H. Rob. & Hatschbach 25152, MBM, Z \\
\hline C. obovata (Less.) Dematt. & Harley $10160, \mathrm{~K}$ \\
\hline C. platensis (Spreng.) H. Rob. & Hatschbach 45949, MBM \\
\hline C. sceptrum (Chodat) Dematt. & Hatschbach 74562, MBM \\
\hline Clibadium armani (Balb.) Sch.Bip. ex O.E. Schulz & D. Sucre 10551, RB \\
\hline Conyza bonariensis (L.) Cronquist & Gluglieri 1620, CGMS \\
\hline C. primulifolia (Lam.) Cuatr. \& Lourteig & Hatschbach 33803, Z \\
\hline C. sumatrensis (Retz.) E.Walker & Oliveira s.n., RB 362548 \\
\hline Cosmos sulphureus Cav. & Spindola 23, CGMS \\
\hline Critonia morifolia (Mill.) R.M. King \& H. Rob. & Malme 2059, S \\
\hline Cyrtocymura cincta (Griseb.) H. Rob. & Hatschbach 49082, MBM, Z \\
\hline C. scorpioides (Lam.) H. Rob. & Hatschbach 60756, MBM \\
\hline Dasyphyllum brasiliense (Spreng.) Cabrera & Malme 2120, S \\
\hline D. sprengelianum (Gardner) Cabrera & Hatschbach 32099, MBM \\
\hline D. vagans (Gardner) Cabrera & Barros 858, SP, UEC \\
\hline Dimerostemma annuит (Hassl.) H. Rob. & Hatschbach 77022, UB \\
\hline D. apense (Chodat) M.D. Moraes & Moraes et al. 631, UEC \\
\hline D. arnottii (Baker) M.D. Moraes & Garcia 14333, UEC \\
\hline D. aspilioides (Griseb.) M.D. Moraes & Rego 698, CGMS \\
\hline D. bishopii H. Rob. & Hatschbach et al. 74386, ESA \\
\hline D. brasilianum Cass. & Hatschbach 33995, MBM \\
\hline D. goyazense (Gardner) M.D. Moraes & Hatschbach 74249, FURB \\
\hline D. grazielae H. Rob. & Hatschbach 35983, NY \\
\hline D. myrtifolium (Chodat) M.D. Moraes & Moraes et al. 619, UEC \\
\hline D. paneroi M.D. Moraes & Moraes 613 , holótipo, UEC; isótipos, NY, RB, SP \\
\hline D. pseudosilphioides (Hassl.) M.D. Moraes & Moraes et al. 630, UEC \\
\hline D. reitzii (H. Rob.) M.D. Moraes & Krapovickas \& Cristóbal 34330, CTES \\
\hline D. retifolium (Sch.Bip. ex Baker) S.F. Blake & Hatschbach $32374, \mathrm{RB}$ \\
\hline D. virgosum $\mathrm{H}$. Rob. & de Paula \& Conceição 1627, COR \\
\hline Disynaphia achillaea (Chodat) R.M. King \& H. Rob. & Hatschbach 46145, MBM \\
\hline D. filifolia (Hassl.) R.M. King \& H. Rob. & Hatschbach 46138, Z \\
\hline D. multicrenulata (Sch.Bip. ex Baker) R.M. King \& H. Rob. & Ribas \& Pereira 2515, ESA \\
\hline D. senecionidea (Baker) R.M. King \& H. Rob. & Hatschbach 25103, MBM \\
\hline Eclipta prostata $(\mathrm{L}.) \mathrm{L}$. & Hatschbach \& Guimarães 22045, RB \\
\hline Elephantopus mollis Kunth & Hatschbach 62337, MBM, Z \\
\hline E. palustris Gardner & Hatschbach 24346, MBM, S \\
\hline E. racemosus Gardner & Ratter $1932, \mathrm{~K}$ \\
\hline E. riparius Gardner & Irwin $17096, \mathrm{~S}$ \\
\hline Eremanthus cinctus Baker & Malme s.n., S \\
\hline Eitenia polyseta R.M. King \& H. Rob. & Cid Ferreira 2555, RB \\
\hline Emilia fosbergii Nicolson & Rodrigues 6, CGMS \\
\hline E. sonchifolia $(\mathrm{L}.) \mathrm{DC}$. & Krapovickas 32816, MBM \\
\hline Enydra anagallis Gardner & Pereira 425, RB \\
\hline Erechtites hieraciifolius (L.) Raf. ex DC. & Conceição 2440, CGMS \\
\hline E. goyazensis (Gardner) Cabrera & Hatschbach 26073, MBM \\
\hline
\end{tabular}


Quadro 1. Cont.

\begin{tabular}{|c|c|}
\hline Espécies & Voucher \\
\hline E. valerianifolius (Wolf) DC. & Damasceno Jr. 2063, CGMS \\
\hline Flaveria bidentis (L.) Kuntze & Hatschbach 29509, MBM \\
\hline Gamochaeta filaginea (DC.) Cabrera & Hatschbach 76258, MBM \\
\hline G. pensylvanica (Willd.) Cabrera & Pott 2779, RB \\
\hline G. stachydifolia (Lam.) Cabrera & Conceição 2534, RB \\
\hline Gymnocoronis spilanthoides DC. & Malme 1768, S \\
\hline Gyptidium trichobasis (Baker) R.M. King \& H. Rob. & Hatschbach 49314, MBM \\
\hline Gyptis alternifolia (Sch. Bip. ex Baker) R.M. King. \& H. Rob. & Hatschbach 74569, MBM \\
\hline G. crassipes (Hieron.) R.M. King \& H. Rob. & Hatschbach 23684, MBM \\
\hline G. lanigera (Hook. \& Arn.) R.M. King \& H. Rob. & Hatschbach 74897, MBM \\
\hline Hebeclinium macrophyllum (L.) DC. & Malme $1408, \mathrm{~S}$ \\
\hline Heterocondylus lysimachioides (Chodat) R.M. King \& H. Rob. & Hatschbach 29444, MBM, Z \\
\hline H. vitalbae (DC.) R.M. King \& H. Rob. & Malme 2086, S \\
\hline Ichthyothere cordata Malme & Maguire, B. \& Maguire, C.K. 44510, NY, RB \\
\hline I. hirsuta Gardner & Passos 1164, HRB, RB \\
\hline I. matogrossensis R.C.A. Pereira \& Semir & Cabrera \& Zardine 30024, holótipo CTES, isótipo LP \\
\hline I. rufa Gardner & Sousa et al. 3205, RB, UB \\
\hline Inulopsis camporum (Gardner) G.L. Nesom & Kuhlmann s.n., RB 2079 \\
\hline Isostigma brasiliense (Gardner) B. D. Jacks. & Riedel 410, P, GH, LP, NY. \\
\hline I. peucedanifolium var. speciosum (Less.) Guad. & Hatschbach 76950 HUEFS. \\
\hline Jungia floribunda Less. & Hatschbach 77308, MBM \\
\hline Koanophyllon simillimum (B.L. Rob.) R.M. King \& H. Rob. & Hatschbach 48535, MBM, Z \\
\hline K. solidaginoides (Kunth) R.M. King \& H. Rob. & Hatschbach 76692, MBM \\
\hline Lagascea mollis Cav. & Hatschbach 74327, MBM \\
\hline Lepidaploa amambaia $\mathrm{H}$. Rob. & Hatschbach 47289, MBM \\
\hline L. psilostachya (DC.) H. Rob. & Hatschbach 74511, MBM \\
\hline L. remotiflora (Rich.) H. Rob. & Hatschbach 21893, MBM \\
\hline \multicolumn{2}{|l|}{ Leptostelma tweediei (Hook. \& Arn.) D.J.N. Hind \& } \\
\hline G.L. Nesom & Damasceno Jr. 4350, CGMS \\
\hline Lessingianthus bardanoides (Less.) H. Rob. & Hatschbach 33933, MBM, Z \\
\hline L. brevifolius (Less.) H. Rob. & Pott $4624, \mathrm{RB}$ \\
\hline L. buddleiifolius (Mart. ex DC.) H. Rob. & Malme $1460, \mathrm{~S}$ \\
\hline L. durus (Mart. ex DC.) H. Rob. & Irwin $16035, \mathrm{~S}$ \\
\hline L. glabratus (Less.) H. Rob. & Irwin 16016, MBM \\
\hline L. grandifloras (Less.) H. Rob. & Malme 2505, S \\
\hline L. mansoanus (Baker) H. Rob. & Malme 2066, S \\
\hline L. mollissimus (D. Don \& Arn. ex Hook.) H. Rob. & Tokarnia $129, \mathrm{RB}$ \\
\hline L. niederleinii (Hieron.) H. Rob. & Hatschbach 62417, MBM, Z \\
\hline L. obscurus (Less.) H. Rob. & Hatschbach 74804, MBM \\
\hline L. onopordioides (Baker) H. Rob. & Malme 1516, S, Z \\
\hline L. platyphyllus (Chodat) H. Rob. & Hatschbach 47315, MBM \\
\hline L. polyphyllus (Sch.Bip. ex Baker) H. Rob. & Hatschbach 58704, MBM \\
\hline L. profusus (Dematteis \& Cabrera) M.B. Ângulo & Garcia 13949, UEC \\
\hline L. psilophyllus (DC.) H. Rob. & Ratter $916, \mathrm{~K}$ \\
\hline L. pumillus (Vell.) H. Rob. & Hatschbach 26116, MBM \\
\hline L. rubricaulis (Humb. \& Bonpl.) H. Rob. & Hatschbach 21951, MBM \\
\hline L. sellowii (Less.) H. Rob. & Hatschbach 58768, MBM \\
\hline L. saltensis (Hieron.) H. Rob. & Malme 3015, S \\
\hline L. varroniifolius (DC.) H. Rob. & Irwin $16424, \mathrm{~S}$ \\
\hline L. virgulatus (Mart. ex DC.) H. Rob. & Malme s.n., $\mathrm{S}$ \\
\hline Lycoseris boliviana Britton & Hatschbach 21906, MBM, RB, MO, NY \\
\hline Melanthera latifolia (Gardner) Cabrera & Hatschbach 73088, MBM \\
\hline Mesanthophora rojasii (Cabrera) H. Rob. & Pott 3645, MBM \\
\hline Mikania capricorni B.L. Rob. & Araújo 92, CGMS \\
\hline M. congesta DC. & Conceição 1439, US \\
\hline M. cordifolia (L.f.) Willd. & Gasparini 2023, RB \\
\hline M. cynanchifolia Hook. \& Arn. ex B.L. Rob. & Pott $1011, \mathrm{RB}$ \\
\hline M. decumbens Malme & Rambo 41433, MOBOT, S \\
\hline
\end{tabular}


Quadro 1. Cont.

\begin{tabular}{|c|c|}
\hline Espécies & Voucher \\
\hline M. laevigata Sch.Bip. ex Baker & Vera s.n., HUFU 53508 \\
\hline M. mendocina Phil. & Hatschbach 71975, MBM \\
\hline M. micrantha Kunth & Hatschbach 73263, MBM \\
\hline M. officinalis Mart. & Cunha 191, CGMS \\
\hline M. parodii Cabrera & Pott 2678, RB \\
\hline M. periplocifolia Hook. \& Arn. & Cid Ferreira 110, RB \\
\hline M. pohliana Sch.Bip. ex Baker & Macedo 3691, RB \\
\hline M. polystachya DC. & Conceição 2013, CGMS, S \\
\hline M. stenophylla W.C. Holmes & Hatschbach 32528, MBM \\
\hline Moquiniastrum barrosoae (Cabrera) G. Sancho & Caliente et al. 1156, HISA \\
\hline M. floribundum (Cabrera) G. Sancho & Hatschbach 45874, MBM, Z \\
\hline M. haumanianum (Cabrera) G. Sancho & Hatschbach 46132, MO \\
\hline M. polymorphum (Less.) G. Sancho & Krapovickas et al. 14033, LP \\
\hline M. ramboi (Cabrera) G. Sancho & Krapovickas et al. 14013, LP \\
\hline Orthopappus angustifolius (Sw.) Gleason & Pereira 51, CGMS \\
\hline Pacourina edulis Aubl. & Cervi 3357, MBM \\
\hline Parthenium hysterophorus L. & Hatschbach et al. 74435, MBM, RB \\
\hline Pectis odorata Griseb. & Almeida 326, RB \\
\hline P. stella Malme & Hatschbach 49120, MBM \\
\hline P. substriata Rusby & Bautista \& Fuks 328, RB \\
\hline P. uniaristata DC. & Hatschbach 36070, MBM \\
\hline Piptocarpha rotundifolia (Less.) Baker & Hatschbach 31864, isótipo NY \\
\hline Pluchea sagittalis (Lam.) Cabrera & Elias de Paula 1885, MBM, Z \\
\hline Porophyllum lanceolatum DC. & Fousek s.n., RB 69439 \\
\hline P. hasslerianum Chodat & Hatschbach 74457, MBM \\
\hline P. oppositifolium (Poir.) DC. & Hatschbach et al. 74805, ASE, ESA, MBM \\
\hline P. ruderale (Jacq.) Cass. & Hatschbach et al. 77334, ALCB \\
\hline Praxelis basifolia (Malme) R.M. King \& H. Rob. & Malme 3198, S \\
\hline P. clematidea (Griseb.) R.M. King \& H. Rob. & Hatschbach 26171, Z \\
\hline P. grandiflora (DC.) Sch.Bip. & Hatschbach 47307, MBM \\
\hline P. insignis (Malme) R.M. King \& H. Rob. & Malme 3477, S \\
\hline P. kleinioides (Kunth) Sch. Bip. & Irwin $16973, \mathrm{~S}$ \\
\hline P. ostenii (B.L. Rob.) R.M.King \& H. Rob. & Hatschbach 34662, MBM, Z \\
\hline P. pauciflora (Kunth) R.M. King \& H. Rob. & Hatschbach 24315, MBM \\
\hline Pseudogynoxys cabrerae H. Rob. \& Cuatrec. & Caliente 1521, HISA \\
\hline Pterocaulon alopecuroides (Lam.) DC. & Melo 4245, HUEFS \\
\hline P. angustifolium $\mathrm{DC}$. & Hatschbach 76904, MBM \\
\hline P. balansae Chodat & Caliente 1876, HISA \\
\hline P. lanatum Kuntze & Cid Ferreira 2038, RB \\
\hline P. lorentzii Malme & Cid Ferreira 1720, RB \\
\hline P. purpurascens Malme & Barbosa 1909, MBM \\
\hline Raulinoreitzia crenulata (Spreng.) R.M. King \& H. Rob. & Hatschbach 49056, MBM, S, Z \\
\hline R. leptophlebia (B.L.Rob.) R.M. King \& H. Rob. & Garcez 20, RB \\
\hline R. tremula (Hook. \& Arn.) R.M. King \& H. Rob. & Hatschbach 77076, MBM \\
\hline Riencourtia oblongifolia Gardner & G. Hatschbach et al. 33026, RB \\
\hline Salmea scandens (L.) DC. & Pott $4909, \mathrm{RB}$ \\
\hline Senecio adamantinus Bong. & Hatschbach 33792, MBM \\
\hline S. apensis Cabrera & Hatschbach 89233, ALCB \\
\hline S. grisebachii Baker & Jacques $728-\mathrm{A}, \mathrm{UB}$ \\
\hline Solidago chilensis Meyen & Cunha 210, CGMS \\
\hline Sphagneticola brachycarpa (Baker) Pruski & Hatschbach 29459, NY \\
\hline S. trilobata (L.) Pruski & Pinto s.n., CGMS 4134 \\
\hline Spilanthes nervosa Chodat & Hatschbach 25018, RB \\
\hline Stenocephalum apiculatum (Mart. ex DC.) Sch.Bip. & Caliente 523, HISA \\
\hline S. hexanthum Sch.Bip. & Hatschbach 76622, MBM \\
\hline S. hystrix (Chodat) H. Rob. & Guerra 80, holótipo RB \\
\hline Stevia alternifolia Hieron. & Hatschbach 38681, MBM, Z \\
\hline S. aristata D.Don ex Hook. \& Arn. & Hatschbach 74395, MBM \\
\hline
\end{tabular}


Quadro 1. Cont.

\begin{tabular}{|c|c|}
\hline Espécies & Voucher \\
\hline S. commixta B.L. Rob. & Hatschbach 23837, MBM \\
\hline S. involucrata Sch.Bip. ex Baker & Hatschbach 58751, MBM \\
\hline S. oligocephala DC. & Hatschbach 77241, MBM \\
\hline S. ophryophylla B.L. Rob. & Hatschbach 23837, MBM \\
\hline S. rebaudiana (Bertoni) Bertoni & Macedo s.n., SP \\
\hline S. rojasii Hassl. & Hatschbach 49309, MBM \\
\hline Stilpnopappus pantanalensis H. Rob. & Cervi 3229, isótipo UPCB \\
\hline S. trichospiroides Mart. & Cervi 3229, MBM \\
\hline Stomatanthes corumbensis (B.L. Rob.) H. Rob. & Smith 94, isótipo NY \\
\hline S. dentatus (Gardner) H. Rob. & Malme $1808, \mathrm{~S}$ \\
\hline S. dictyophyllus (DC.) H. Rob. & Hatschbach et al.101694, BHCB \\
\hline S. subcapitatus (Malme) H. Rob. & Sano $217, \mathrm{MO}$ \\
\hline S. trigonus (Gardner) H. Rob. & Malme $2404, \mathrm{~S}$ \\
\hline Strophopappus bullatus (Krasch.) Sch.Bip. ex R. Esteves & Hatschbach 31933, MBM \\
\hline Symphyotrichum squamatum (Spreng.) G.L. Nesom & Fiori s.n., FUEL 2872 \\
\hline Tagetes minuta $\mathrm{L}$. & Conceição 1905, CGMS \\
\hline Tilesia baccata (L.) Pruski & Guimarães 1377, RB \\
\hline Trichogonia hassleri Mattf. & Hatschbach et al. 74603, HUEFS, US \\
\hline T. salviifolia Gardner & Hatschbach et al. 76222, SPF, US \\
\hline Trichospira verticillata (L.) S.F. Blake & Hatschbach 63743, MBM, Z \\
\hline Tridax procumbens L. & Hatschbach 52492, MBM \\
\hline \multicolumn{2}{|l|}{ Trixis antimenorrhoea (Schrank) Kuntze subsp. } \\
\hline antimenorrhoea & Hatschbach 74610, ALCB \\
\hline T. pallida Less. & Hatschbach 74271, MBM \\
\hline T. verbasciformis Less. & Hatschbach 76940, MBM \\
\hline Urolepis hecatantha (DC.) R.M. King \& H. Rob. & Dubs $1269, \mathrm{~K}, \mathrm{Z}$ \\
\hline Verbesina sordescens DC. & Hatschbach 77125 (MBM) \\
\hline Vernonanthura brasiliana (L.) H. Rob. & Cook et al. $591, \mathrm{Z}$ \\
\hline V. catharinensis (Cabrera) H. Rob. & Reitz 10834, MBM \\
\hline V. chamaedrys (Less.) H. Rob. & Hatschbach 46111, MBM \\
\hline V. cuneifolia (Gardner) H. Rob. & Hatschbach 32514, MBM \\
\hline V. ferruginea (Less.) H. Rob. & Hatschbach 21781, MBM, Z \\
\hline V. ignobilis (Less.) H. Rob. & Malme $2413, \mathrm{~S}$ \\
\hline V. mariana (Mart. ex Baker) H. Rob. & Hatschbach 33037, Z \\
\hline V. membranacea (Gardner) H. Rob. & Anderson 9726, MBM \\
\hline V. mucronulata (Less.) H. Rob. & A.J. s.n., RB 363231 \\
\hline V. oligactoides (Less.) H. Rob. & Hatschbach 76946, MBM \\
\hline V. oligolepis (Sch.Bip. ex Baker) H. Rob. & Hatschbach 58584, MBM \\
\hline$V$. petiolaris (DC.) H. Rob. & Pott et al. 2406 \\
\hline$V$. polyanthes (Sprengel) Vega \& Dematteis & Jacques 1737, CGMS \\
\hline V. tweediana (Baker) H. Rob. & Krapovickas s.n., MBM \\
\hline Vernonia gertii Dematt. & Hatschbach 72950, holótipo MBM, isótipos ALCB, MBM, RB \\
\hline$V$. pottii R. Esteves & Pott $11026, \mathrm{MBM}$ \\
\hline Wedelia brachylepis Griseb. & Hatschbach 67562, MBM \\
\hline W. hatschbachii H. Rob. & Hatschbach 33047, NY \\
\hline W. kerrii N.E. Br. & Hatschbach 46143, MBM \\
\hline W. subvelutina DC. & Barbosa 1424, RB \\
\hline W. trichostephia DC. & Ribas 2606, MBM \\
\hline Zinnia elegans Jacq. & Wachter s.n., ALCB 105984 \\
\hline
\end{tabular}

\section{Principais Acervos}

Embora nem todas as coleções dos herbários no Brasil estejam representadas em sua totalidade no CRIA (2017), observa-se que os principais acervos em Asteraceae, até o momento, são citados para o Jardim Botânico do Rio de Janeiro (RB 44.460 espécimes), Herbário do Museu
Botânico Municipal de Curitiba (MBM 31.118) e Herbário de Instituto de Ciências Naturais da Universidade do Rio Grande do Sul (ICN 17.414), seguidos pelo Herbário da Universidade Federal de Uberlândia (HUFU 14.245) e Herbário da Universidade Federal de Minas Gerais (BHCB 12.167). Cabe destacar que os maiores acervos na família 
estão diretamente relacionados ao tamanho geral da coleção, à presença de especialistas nestas instituições, como a Dra. Graziela Maciel Barroso no Jardim Botânico do Rio de Janeiro, ou pela presença de renomados coletores, como Gert Hatschbach (MBM).

Quanto aos acervos com maior número de espécimes em Asteraceae provenientes do estado, o Herbário da Universidade Federal do Mato Grosso do Sul (CGMS) é o com maior registro (2.478 espécimes), seguido pelos Herbários do Museu Botânico Municipal (MBM 517) e Jardim Botânico do Rio de Janeiro (RB 404). Destes, o CGMS apresenta 753 espécimes identificados até gênero, com destaque para Vernonia Schreb (83), Eupatorium L. s.l. (63) e Mikania Willd. (54), seguido pelo RB (44 espécimes identificados até gênero) e, finalmente, o MBM, onde $100 \%$ dos espécimes estão identificados.

Desses resultados, cabe destacar que o elevado número de espécimes não identificados no herbário CGMS pode ser reflexo da carência de especialista ou estudante trabalhando exclusivamente com Asteraceae no estado. Por outro lado, os herbários que recebem um maior número de visitantes especialistas ou cujo curador tem uma política que fortalece o intercâmbio de materiais com outros herbários nacionais ou internacionais, apresentam o menor número de táxons indeterminados, como os Herbários do Museu Botânico Municipal (MBM) e o Jardim Botânico do Rio de Janeiro (RB).

\section{Perspectivas de Pesquisa para o Grupo nos próximos 10 anos}

A partir dos dados disponibilizados em rede, destacamos que o Cerrado s.l. é o principal domínio para a concentração de estudos exploratórios (inventários) em Asteraceae e, dentre as regiões geográficas com maiores lacunas, destacam-se o Tocantins, Goiás, Mato Grosso do Sul e Mato Grosso. Sendo assim, espera-se que nos próximos anos haja um esforço da comunidade científica no Brasil em aumentar projetos de levantamentos florísticos em áreaschave do país, buscando subsídios para o conhecimento e a conservação da biodiversidade.

\section{AGRADECIMENTOS}

Os autores agradecem ao Projeto REFLORA (Proc. 563541/2010-5) pelo apoio financeiro aos estudos de Asteraceae no Brasil. Ao Conselho Nacional de Desenvolvimento Científico e Tecnológico pelas bolsas de Iniciação Científica e bolsa Pesquisador concedida à primeira autora e à Coordenação de Aperfeiçoamento de Pessoal de Nível Superior pelas bolsas de pós-doutorado concedidas ao primeiro, segundo e último autores.

\section{REFERÊNCIAS}

Barroso, G.M. 1976. Asteraceae-subtribo Baccharidinae Hoffmann: estudo das espécies ocorrentes no Brasil. Rodriguésia 28(40): 3-273.

Bautista, H.P. 1986. Pectis L. (Asteraceae, Tageteae). Espécies ocorrentes no Brasil. Arquivos do Jardim Botânico do Rio de Janeiro 27: 20-107.
Blake, S.F. 1921. Revision of the genus Acanthospermum. Contributions from the United States National Herbarium 20: 383-392.

Bolick, M.R. 1991. Systematics of Salmea DC. (Asteraceae: Heliantheae). Systematic Botany 16(3): 462-477.

Brazil Flora Group BFG. 2015. Growing knowledge: an overview of Seed Plant diversity in Brazil. Rodriguésia 66(4): 1085-1113.

Bringel JR., J.B.A. 2007. A tribo Heliantheae Cassini (Asteraceae) na bacia do rio Paranã (GO, TO). Dissertação 166 f.. Universidade de Brasília, Brasília.

Cabrera, A.L. 1957. El genero Senecio (Asteraceae) en Brasil, Paraguay y Uruguay. Arquivos do Jardim Botânico do Rio de Janeiro 15: 163-264.

Cabrera, A. L. 1971. Revisión del género Gochnatia (Asteraceae). Revista del Museo de La Plata, Sección Botánica 12: 1-160.

Canne, J.N. 1977. A revision of the genus Galinsoga (Compositae: Heliantheae). Rodhora 79: 319-389.

Centro de Referência em Informação Ambiental - CRIA. 2017. SpeciesLink. Disponível em: http://www.splink.org.br/index . Acessado em 05.05.2017

Deble, L.P. 2007. O gênero Achyrocline (Less.) DC. (Asteraceae, Gnaphalieae) no Brasil. Tese 134 f., Universidade Federal de Santa Maria, Rio Grande do Sul.

Dubs, B. 1998. Prodromus Florae Matogrossensis. The Botany of Mato Grosso. Küsnacht: Betrona, Series B 3: 60-73.

Flora Integrada da Região Centro-Oeste - Florescer 2017. Disponível em: http://www.florescer.unb.br/. Acessado em 05.05.2017

Funk, V.A., Susanna, A., Stuessy, T.F. \& Robinson, H. 2009. Classification of Asteraceae. In Systematics, Evolution and Biogeography of the Asteraceae (V.A. Funk, A. Susanna, T.F. Stuessy \& R.J. Bayer, eds.) IAPT, Vienna, p.171-189.

Hind, D.J.N. \& Miranda, E.B. 2008. Lista Preliminar da família Compositae. Royal Botanic Gardens, Kew. 104 p.

International Union for Conservation of Nature - IUCN 2010. Red List of Threatened Species. Version 2010.4. Disponível em: www.iucnredlist. org. Acessado em 13.05.2015.

Jansen, R.K. 1981. Systematics of Spilanthes (Asteraceae: Heliantheae). Systematic Botany 6(3): 231-257.

Jansen, R.K. 1985. The Systematics of Acmella (Asteraceae-Heliantheae). Systematic Botany Monographs 8: 1-115.

Magenta, M.A.G. 2006. Viguiera Kunth (Asteraceae, Heliantheae) na América do Sul e sistemática das espécies do Brasil. Tese 339 f., Universidade de São Paulo, São Paulo.

Mendonça, R.C., Felfili, J.M., Walter, B.M.T., Silva-Júnior, M.C., Rezende, A.V., Filgueiras, T.S., Nogueira, P.E. \& Facg, C.W. 2008. Flora vascular do bioma Cerrado: um checklist com 12.356 espécies. In Cerrado: ambiente e ecologia (S.M.Sano, S.P. Almeida \& J,F. Ribeiro, eds.). Embrapa Informação Tecnológica, Brasília, v.2, p.421-1279.

Mondin, C.A. 2004. Levantamento da tribo Heliantheae Cass. (Asteraceae), sensu stricto, no Rio Grande do Sul, Brasil. Tese 375 f., Universidade Federal do Rio Grande do Sul, Rio Grande do Sul.

Moraes, M.D. \& SEMIR, J. 2009. A revision of Brazilian Dimerostemma (Asteraceae, Heliantheae, Ecliptinae), with a new species and taxonomic adjustments. Brittonia 61(4): 341-365.

Panero, J.L. \& Funk, V.A. 2008. The value of sampling anomalous taxa in phylogenetic studies: Major clades of the Asteraceae revealed. Molecular Phylogenetics and Evolution 47: 757-782.

Pereira, R.C.A. 2001. Revisão Taxonômica do gênero Ichthyothere Mart. (Heliantheae-Asteraceae). Tese 211 f., Universidade Federal Rural de Pernambuco, Recife.

Powell, M.A. 1965. Taxonomy of Tridax (Compositae). Brittonia 1(17): 47-96.

Roque, N. \& Bautista, H. 2008. Asteraceae: Caracterização e Morfologia Floral. Universidade Federal da Bahia, Salvador. 79 p.

Saavedra, M.M. 2011. Sistemática de Dasyphyllum (Asteraceae). Tese_247_f., Instituto de Pesquisas Jardim Botânico do Rio de Janeiro, Rio de Janeiro.

Santos, J.U.M. 2001. O gênero Aspilia Thou. (Asteraceae - Heliantheae) no Brasil. Museu Paraense Emílio Goeldi, Belém. 301 p.

Sherff, E.E. 1932. Revision of the genus Cosmos (family Asteraceae). Field Museum Publications in Botany 8: 401-447. 
Sherff, E.E. 1937. The genus Bidens, I \& II. Field Museum of Natural History, Botanical Series 16: 1-709.

Stuessy, F. 1972. Revision of the Genus Melampodium (Compositae: Heliantheae). Rhodora 74: 1-70.

Teles, A.M, Loeuille, B., Hattori, E.K.O., Heiden, G., Bautista, H.P., Grokoviski, L., Ritter, M.R., Saavedra, M.M., Roque, N., Borges, R.A.X. \& Liro, R.M. 2009. Asteraceae. In Plantas da Floresta
Atlântica (J.R. Stehmann, R.C. Forzza, M. Sobral, A. Salino \& L.H.Y. Kamino, orgs.). Jardim Botânico do Rio de Janeiro, Rio de Janeiro, p.150-173.

Urtubey, E. 1999. Revisión del gênero Barnadesia (Asteraceae: Barnadesioideae, Barnadesieae). Annals of Missouri Botanical Garden 86: 57-117. 